\title{
„Gesund - erfolgreich - gut? Zur Diskussion gerontologischer und politischer Leitbilder des Alterns“
}

Nürnberg, 10.-11. Oktober 2019

\author{
Nadja Morgenstern • Tobias Schramm
}

Online publiziert: 16. März 2020

(C) Der/die Autor(en) 2020

In Konzepten des „,successful aging“ kommt der Anspruch einer Überwindung lange vorherrschender Defizitmodelle des Alterns zu Gunsten einer an Ressourcen und Potenzialen orientierten Perspektive auf das spätere Leben zum Tragen. Sie stehen damit für ein zentrales Paradigma zeitgenössischer human- und sozialwissenschaftlicher Alternsforschung und finden auch Eingang in medial vermittelte öffentliche Diskurse sowie sozial- und gesundheitspolitische Programme in den alternden Gesellschaften der Gegenwart. Dabei spiegeln sie nicht nur sich wandelnde Erfahrungen des Älterwerdens und Altseins wider, sondern bringen auch individuelle und gesellschaftliche Leitbilder für das höhere Lebensalter zur Geltung. Was ist die Funktion dieser gerontologischen und politischen Leitbilder des Alterns? Und sind diese Leitbilder überhaupt sinnvoll und akzeptabel? Welche Rolle spielt Gesundheit? Vor dem Hintergrund dieser Fragen fand am 10. und 11. Oktober 2019 in Nürnberg die interdisziplinäre Abschlusstagung des vom Bundesministerium für Gesundheit geförderten Projektes ,Erfolgreiches = gesundes = gutes Altern? Altersbilder und Prämissen gerontologischer und sozialpolitischer Altersdiskurse in Deutschland“ (2017-2019) statt. Organisiert wurde die Tagung von Dr. Larissa Pfaller, Soziologin an der Friedrich-Alexander-Universität Erlangen-Nürnberg (FAU), und Prof. Dr. Mark Schweda, Philosoph und Medizinethiker an der Carl von Ossietzky Universität Oldenburg in Zusammenarbeit mit PD Dr. Lars Allolio-Näcke vom Zentralinstitut für Anthropologie der Religionen (FAU). Moderiert wurden die Einzel- und Doppelvorträge und die anschließenden Diskussionen mit insgesamt zwölf Referent*innen von Lena Stange, Dr. Merle Weßel und Niklas Ellerich-Groppe (Carl von Ossietzky Universität Oldenburg). Ergänzt wurde die Veranstaltung durch Posterbeiträge von Alexandra Sept

\footnotetext{
N. Morgenstern · T. Schramm $(\bowtie)$

Institut für Soziologie, Friedrich-Alexander-Universität Erlangen-Nürnberg,

Kochstraße 4, 91054 Erlangen, Deutschland

E-Mail: tobias.schramm@fau.de
} 
(München), Niklas Petersen (Jena), Merle Weßel und Lena Stange (Oldenburg), Lisa Häberlein (Erlangen), Marina Plugge (Köln), Luise Geithner und Marcella Reissmann (Köln), sowie Katrin Ettl (Erlangen).

\section{Empirische und ethische Perspektiven des „Successful Aging““}

Larissa Pfaller und Mark Schweda begrüßten die Teilnehmer*innen und Zuhörer*innen und erörterten, warum sich Soziolog*innen und Ethiker*innen überhaupt mit dem Konzept des ,erfolgreichen Alterns“ beschäftigen und warum genau dieser Begriff eine interdisziplinäre Zusammenarbeit von Philosoph*innen und Soziolog*innen erfordert. Pfaller und Schweda legten dar, dass das Konzept des ,erfolgreichen Alterns" einen moralisch aufgeladenen Begriff darstellt, dessen evaluative und normative Prämissen einer eigenen Untersuchung unterzogen werden müssen.

Den Eröffnungsvortrag hielt Prof. Dr. Dr. h.c. Heiner Bielefeldt, der an der FAU den Lehrstuhl für Menschenrechte und Menschenrechtspolitik innehat. Der Vortrag widmete sich der Bedeutung der Ausformulierung von Menschenrechten für Ältere. Bielefeldt stellte die einleitende Frage, ob es überhaupt sinnvoll ist, spezielle Menschenrechte für Ältere zu bestimmen und in einer eigenen Charta auszuformulieren. Menschenrechte seien universell gültig, müssen aber für spezifische Kontexte konkretisiert werden. Lebensweltliche Perspektiven verliehen Menschenrechten überhaupt erst einen konkreten Sinn. Nur wenn solche spezifischen Perspektiven eingenommen würden, könne auch auf neue Problembereiche und Rechtsdefizite reagiert werden. Die eingenommen Perspektiven fragmentierten die Menschenrechte nicht, sondern bereicherten unser Verständnis dessen, was ein Menschenrecht überhaupt ist.

Im ersten Vortrag der Tagung stellten Larissa Pfaller und Mark Schweda die Ergebnisse ihrer Untersuchung des Konzeptes des ,erfolgreichen Alterns“ vor. Dieses wird in der gerontologischen Literatur als dominantes Konzept und Leitmotiv einer „,neuen Gerontologie“ verstanden. Zugleich ist das Konzept massiver Kritik ausgesetzt. Vor diesem Hintergrund stellt sich die Frage: Warum ist der Begriff des ,erfolgreichen Alterns“ derart allgegenwärtig und erfolgreich? Aus Experteninterviews mit Gerontolog*innen konnte die Einsicht gewonnen werden, dass dieses Konzept lediglich für kleine Teile der gerontologischen Psychologie tatsächlich als operatives Konzept dient. Außerdem wird in den einschlägigen Debatten letzten Endes immer auch die Frage erörtert, wie wir alt werden sollen. Damit weist die Diskussion über den gerontologischen Fachdiskurs hinaus auf die ethische Fragestellung, was ein gutes, gelingendes Leben im Alter sei. In ihrem Licht erscheint die Debatte allerdings vielfach verkürzt. So weist sie z.B. eine einseitige Ausrichtung auf Aktivität auf. Insgesamt kann die ethische Analyse der normativen Prämissen des erfolgreichen Alterns eine aktivistische, produktivistische und ökonomistische Reduktion des Begriffs offenlegen.

Im zweiten Vortrag der ersten Tagungssektion lieferte die Soziologin Marie-Kristin Döbler (FAU) mit ihrem Vortrag empirische Perspektiven zur „Lebenszufriedenheit in Alten(pflege)heimen“ und zur Frage, was „gutes Leben“ für alte Menschen bedeutet. Entsprechend lag der Fokus auf dem feldimmanenten Verständnis von gu- 
tem Leben. Die Pflegepraxis scheint von der Idee eines ,guten Alterns“ geprägt zu sein, die aber nicht unbedingt mit der Vorstellung eines aktiven, produktiven und erfolgreichen Alterns einhergeht. Stattdessen können alternative Verständnisse von Aktivität und somit eine Pluralität von Aktivitäten gefunden werden, die über die aktivistischen Engführungen der gerontologischen Debatte hinausweisen.

Im Anschluss setzten sich Susanne Wurm und Miranda Leontowitsch in einer gemeinsamen Diskussionsrunde mit der Frage auseinander, welche Rolle Gesundheit in den Leitbildern des Alterns spielt und ob das Leben ohne Gesundheit nicht mehr als lebenswert erscheint. Prof. Dr. Susanne Wurm (Universität Greifswald), die einen Lehrstuhl für Sozialmedizin und Prävention innehat, fragte in ihrem Vortrag, was wir überhaupt unter Gesundheit verstehen und welche Bedeutung diese für uns hat. Sie stellte dabei insbesondere die Bedeutung von Altersbildern für das individuelle Gesundheitsverhalten und die Gesundheitsversorgung im Alter heraus. In diesem Zusammenhang plädierte sie für ein vielschichtigeres Gesundheitssystem, welches verschiedene Anforderungen an ein ausdifferenziertes Verständnis von Gesundheit erfüllen könnte.

Der Vortrag der Soziologin Dr. Miranda Leontowitsch (Universität Frankfurt am Main) befasste sich unter dem Titel „Ohne Gesundheit ist alles nichts?“ mit der Kunst des Alterns. Nach Leontowitsch sind Gesundheit und der Umgang mit gesundheitlichen Risiken zunehmend berechenbar geworden. Gesundheit sei zu einer individuellen moralischen Aufgabe geworden. Umso wichtiger erscheint Leontowitsch vor diesem Hintergrund die Selbstsorge der Alternden, die als ,,individuelle Handlungsfähigkeit“ zu verstehen ist. Leitbilder des ,,aktiven und erfolgreichen Alter(n)s“ seien nicht Auslöser, sondern Ergebnis von gesellschaftlichen Entwicklungen, die dazu geführt haben, dass Gesundheit und Selbstsorge zur Kernaufgabe des Alter(n)s wurden.

Der gemeinsame Abendvortrag der Gerontologen Prof. Dr. Hans-Werner Wahl (Universität Heidelberg) und Prof. Dr. Clemens Tesch-Römer (Deutsches Zentrum für Altersfragen, Berlin) setzte sich unter dem Titel „Erfolgreiches Altern und die dunkle Seite des Älterwerdens“ mit dem „Ringen der Gerontologie“ um ein Verständnis von Sinnfindung im Alter auseinander. Zudem wurde die Frage beleuchtet, inwiefern das Konzept des ,erfolgreichen Alterns“ weiterhin verwendet werden sollte. Die Gerontologie könne nach Wahl und Tesch-Römer durchaus auf eine Erfolgsgeschichte des ,Successful Aging“ zurückblicken, das sich als Konzept auch noch weiterentwickle. Ihnen zufolge sollte das umstrittene Konzept auch weiterhin verwendet werden, da es visionär sei und impliziere, dass Altern nicht nur unabänderliches biologisches Schicksal, sondern auch durch Interventionen modifizierbar sei. Zudem sei gerade die Normativität des Konzeptes nützlich, da uns so Kriterien dafür an die Hand gegeben werden, worin ,erfolgreiches Altern“ eigentlich bestehe.

\section{Kritische Betrachtungen zum Konzept des „Successful Ageing“}

Im ersten Vortrag des zweiten Tages stellte der Philosoph PD Dr. Magnus Schlette (Universität Heidelberg) die Frage, was unter „Selbstverwirklichung im Alter“ zu verstehen ist. Die Überlegungen zum Konzept der Selbstverwirklichung orientieren 
sich am modernen Freiheitsbegriff und seiner Verbindung mit dem Begriff des Guten. Die Verschränktheit von personaler Freiheit und gutem Leben könne über den Zusammenhang von „Wollen“ und „Können“ verständlich gemacht werden. Selbstverwirklichung müsse vom Willen ausgehen, der das Gute anstrebt. Freude und das Gute finden wir aber schließlich in der Ausübung unserer Fähigkeiten. Wir verwirklichen uns durch unseren Willen im Handeln - in dem, was wir „gut“ können.

Im Anschluss beschäftigte sich der Soziologe Dr. Ludwig Amrhein (Fachhochschule Dortmund) in seinem Vortrag „Kritik der Kritischen Gerontologie“ mit der Frage, was kritische Gerontologie eigentlich ausmacht. Dabei ging Amrhein zunächst auf die von der Kritischen Theorie geprägten Grundlagen und Methoden dieser gerontologischen Strömung ein. Vor diesem Hintergrund skizzierte er, welche inhaltlichen Einwände die kritische Gerontologie gegenüber traditionellen gerontologischen Ansätzen erhebt. Die kritische Gerontologie müsse aber auch sich selbst und ihre eigenen Konzepte und Ansätze kritisch hinterfragen. Sie sei ein soziales Unternehmen, das selbst wiederum ideologiekritisch beleuchtet werden sollte.

In der zweiten Diskussionsrunde der Tagung setzten sich Klaus Schroeter und Heinz Rüegger unter dem Titel „Gelingendes Altern - Lebenskunst oder neoliberaler Imperativ?“ mit der Frage nach dem erfolgreichen Altern auseinander. Der Soziologe Prof. Dr. Klaus Schroeter (Fachhochschule Nordwestschweiz) legte den Schwerpunkt auf die Frage, inwiefern das ,erfolgreiche Altern“ als gesellschaftlicher Imperativ angesehen werden kann. Sein praxeologischer Altersbegriff ermöglicht durch die Untersuchung alltäglicher Handlungen im späteren Lebensalter einen Zugang zur Konstruktion von Altersdifferenzen. Altern verweist, so Schroeter, immer auf einen symbolischen Zusammenhang und wird praktisch vor dem Hintergrund gesellschaftlicher Machtverhältnisse geschaffen. Das Altern und dessen Leitbilder und die damit verbunden kleinen Altershandlungen erschaffen und instituieren sich im gesellschaftlichen Handeln.

Der Vortrag des Theologen Dr. Heinz Rüegger (Institut Neumünster) befasste sich unter dem Titel „Gelingendes Altern als Lebenskunst“ mit der Kunst des Alterns. Rüegger skizzierte zunächst, welche grundlegenden Imperative einer Kunst des Alterns eigen sind. Entgegen den gesellschaftlich vorherrschenden Imperativen, dass das Altern möglichst vermieden oder hinausgezögert bzw. verschleiert werden sollte, gehe es der Lebenskunst des Alterns darum, Potenziale des Alterns zu nutzen. Das Konzept des ,erfolgreiches Alterns“ und die Lebenskunst des Alterns seien insofern $\mathrm{zu}$ unterscheiden, da Letztere nicht produktivistisch oder aktivistisch ausgelegt sein muss, sondern sich auch in der (z. B. kontemplativen) Bewältigung von negativen Alternsprozessen äußere. Die Lebenskunst suche nach Praktiken und Methoden, mit den Herausforderungen des Alterns umzugehen.

\section{Fazit}

In der Abschlussdiskussion der Tagung wurden im Plenum entscheidende Diskussionsstränge und Ergebnisse zusammengefasst: Zunächst wurde noch einmal die Funktion des Konzeptes „Successful Aging“ für den gerontologischen Fachdiskurs thematisiert. Trotz aller - ja auch sehr nachdrücklicher und grundsätzlicher - Kritik 
hält die Gerontologie weiterhin an den Konzepten bzw. aus forschungs- und veröffentlichungspragmatischen Gründen am bloßen Wording "Successful Aging“ fest. Zudem wurden über die Tagung getragene Diskussionsstränge zum Thema Normativität und Gesundheit zusammengeführt: Nicht selten wird an Konzepten des „Successful Aging" eine Kryptonormativitiät kritisiert, also ein expertokratisches Setzen von Normen und Werten, deren Maßstäbe allerdings unausgewiesen bleiben. Ihr wird gleichzeitig als gleichsam ausgleichende Größe die Praxis im Alltag der „Betroffenen" gegenübergestellt. Begreift man allerdings Leitbilder des erfolgreichen Alterns eher als Ergebnis denn als Ursache größerer gesellschaftlicher Entwicklungen, der Medikalisierung und Responsibilisierung des Alter(n)s, ist die Frage nach dem Verhältnis von Betroffenen- und Expertenperspektive noch einmal differenzierter zu stellen. Schließlich sind nicht nur die normativen Prämissen von Konzepten erfolgreichen Alterns selbst, sondern auch die ihrer Kritiker*innen zu hinterfragen.

Funding Open Access funding provided by Projekt DEAL.

Open Access Dieser Artikel wird unter der Creative Commons Namensnennung 4.0 International Lizenz veröffentlicht, welche die Nutzung, Vervielfältigung, Bearbeitung, Verbreitung und Wiedergabe in jeglichem Medium und Format erlaubt, sofern Sie den/die ursprünglichen Autor(en) und die Quelle ordnungsgemäß nennen, einen Link zur Creative Commons Lizenz beifügen und angeben, ob Änderungen vorgenommen wurden.

Die in diesem Artikel enthaltenen Bilder und sonstiges Drittmaterial unterliegen ebenfalls der genannten Creative Commons Lizenz, sofern sich aus der Abbildungslegende nichts anderes ergibt. Sofern das betreffende Material nicht unter der genannten Creative Commons Lizenz steht und die betreffende Handlung nicht nach gesetzlichen Vorschriften erlaubt ist, ist für die oben aufgeführten Weiterverwendungen des Materials die Einwilligung des jeweiligen Rechteinhabers einzuholen.

Weitere Details zur Lizenz entnehmen Sie bitte der Lizenzinformation auf http://creativecommons.org/ licenses/by/4.0/deed.de.

Interessenkonflikt N. Morgenstern und T. Schramm geben an, dass kein Interessenkonflikt besteht. 